\title{
Next generation drug-eluting stents: focus on bioabsorbable platforms and polymers
}

This article was published in the following Dove Press journal:

Medical Devices: Evidence and Research

9 November 2009

Number of times this article has been viewed

\section{Brendan Doyle David R Holmes Jr \\ Division of Cardiovascular Diseases, Department of Internal Medicine, Mayo Clinic College of Medicine, Rochester, Minnesota, USA}

\begin{abstract}
The success of drug-eluting stents in preventing restenosis has shifted the focus of new stent development toward enhancing long term safety and efficacy of these devices, while simultaneously eliminating the need for indefinite dual antiplatelet therapy. A technical advance fulfilling these aims would hold tremendous potential to reduce morbidity, mortality and economic costs associated with the percutaneous treatment of coronary artery disease. An attractive approach is the use of bioabsorbable stent designs. These may include stents with different bioabsorbable drugs, bioabsorbable polymers or even bioabsorbable metallic backbones. A device that could achieve excellent acute and long-term results, but disappear completely within months (thereby avoiding the need for prolonged dual antiplatelet therapy), would be a tremendous advance. Too good to be true? We explore here the scientific rationale and prospects for success with this exciting concept.
\end{abstract}

Keywords: percutaneous coronary intervention, biodegradable, bioabsorbable, polymer, stent

\section{Introduction}

The success of drug-eluting stents (DES) in preventing restenosis has shifted the focus of new stent development toward enhancing long term safety and efficacy of these devices, whilst simultaneously eliminating the need for indefinite dual antiplatelet therapy. A technical advance fulfilling these aims would hold tremendous potential to reduce morbidity, mortality and economic costs associated with the percutaneous treatment of coronary artery disease.

Myocardial infarction (MI) occurring late after percutaneous coronary intervention (PCI) with DES is a complex problem. Numerous mechanisms have been described which may be responsible for acute thrombotic occlusion within the treated segment. Those cited most often include hypersensitivity reactions to the drug/polymer coating, inadequate endothelialization of the stent, and aneurysmal remodeling of the vessel wall leading to stent malapposition. Although these processes are biologically distinct, the end result is the same; the risk for generation of a localized thrombogenic milieu which may not resolve, due to the permanent presence of the metallic platform and polymer.

In this context, the role of dual antiplatelet therapy in contemporary practice has become a contentious issue. Although current recommendations for prolonged use of aspirin and a thienopyridine were directed to address some of the safety problems which have been highlighted, the efficacy of this approach has yet to be proven in randomized trials. Furthermore, this strategy increases the long-term bleeding risk
Mayo Clinic, 200 First St SW, Rochester

MN 55905, USA

$\mathrm{Tel}+\mathrm{I} 507-2552504$

Fax + I 507-2552550

Email holmes.david@mayo.edu 
and expense associated with PCI, as well as complicating subsequent medical care (such as the need for invasive surgical procedures, or co-existing requirements for anticoagulation with warfarin). The emerging issue of antiplatelet drug resistance is yet another concern, although the true extent to which this phenomenon exists and contributes to late adverse events following PCI is still unknown.

An attractive approach to solving many of these problems is the concept of bioabsorbable stent designs. These may include stents with different bioabsorbable drugs, bioabsorbable polymers or even bioabsorbable metallic backbones. Such a device that could achieve excellent acute and longterm results, but disappear completely within months and avoid the need for prolonged dual antiplatelet therapy, would be a tremendous advance. Too good to be true? We explore here the scientific rationale and prospects for success with this exciting concept.

\section{Late myocardial infarction after $\mathrm{PCl}$}

Reports of late thrombosis of DES after these devices were approved for use in routine clinical practice prompted a major controversy about their safety. Evidence of incomplete re-endothelialization, aneurysmal vessel wall remodeling and local hypersensitivity reactions in DES-treated vessels suggested that impaired post-intervention healing of the vessel wall may provide a substrate for late (30 days to 1 year) and very late ( $>1$ year) thrombotic events. ${ }^{1-5}$ Bare metal stents (BMS) do not possess an anti-proliferative coating and although the risk of restenosis is higher with BMS, this was traditionally perceived as a relatively benign complication. ${ }^{6-8}$ This led to a resurgence in use of BMS at the height of the DES thrombosis controversy. Such assumptions regarding the benign nature of restenosis would tend to support an approach to future stent development centered upon refinement of the drug-polymer combination, rather than a fundamental re-evaluation of the need for a durable metallic platform.

Such intense focus on safety led ultimately to a number of important new perspectives on late adverse events, and how these may relate to the index procedure and the kind of device that was used. In particular, the concepts that (1) late stent thrombosis did not occur with BMS and (2) that BMS restenosis was benign was examined in a study of 4503 patients treated with BMS and dual antiplatelet therapy at the Mayo Clinic between 1994 and 2000. ${ }^{9}$ The cumulative incidence of stent thrombosis was $0.5 \%$ at 30 days (95\% confidence interval [CI]; $0.3 \%-0.7 \%$ ), $0.8 \%$ at 1 year $(95 \% \mathrm{CI} ; 0.6 \%-1.1 \%)$ and $2.0 \%$ at 10 years $(95 \% \mathrm{CI}$;
$1.5 \%-2.5 \%$ ). Risk of late (30 days to 1 year) and very late $(>1$ year) BMS thrombosis was increased among patients considered 'off label' for BMS use $(P=0.024)$. Mortality was markedly increased following late and very late BMS thrombosis, particularly during the first 30 days (hazard ratios: 22 [95\% CI 3.1-159] and 40 [95\% CI 15-107] respectively). The 10 year incidence of clinical restenosis was $18.1 \%$ (95\% CI; 16.5\%-19.7\%); many of these patients presented with an acute coronary syndrome and some presented with MI in 2.1\% (95\% CI; 1.6\%-2.6\%). Restenosis presenting with MI was associated with increased mortality compared to no restenosis (hazard ratio $2.37, P<0.001$ ) and to restenosis with a non-MI presentation (hazard ratio 2.42, $P<0.001$ ).

The implications of these findings for future stent development are important, as they introduce doubt about the notion that safety can be enhanced (and the need for indefinite dual-antiplatelet therapy avoided) simply by modifying the drug-polymer coating on the bare metal stent platform. The big question is whether the long-term presence of the stent platform itself is actually a help or sometimes a hindrance in our efforts to improve outcomes. As technology in biodegradable materials has evolved, the potential to manufacture a drug-eluting stent that is partially or completely bioabsorbable has now taken center stage as a novel and extremely exciting therapeutic concept.

\section{Eliminating the permanent polymer}

The first generation of polymer-based DES rapidly replaced bare metal stents as the treatment of choice for percutaneous coronary revascularization. However, the long-term safety of polymer-based DES has been called into question because of concerns about late stent thrombosis secondary to impaired arterial healing, characterized by delayed re-endothelialization and persistence of fibrin. ${ }^{2,7,10}$ Emerging evidence suggests that drug delivery polymers may play an important role in the pathophysiology of impaired healing by provoking inflammatory cell infiltration and/or causing long-term drug sequestration within the arterial wall. ${ }^{5,11,12}$ In addition, there are some data to indicate that the use of polymer-based DES for interventions involving multiple stents (such as bifurcations or overlapping stents) may further exacerbate local arterial toxicity, which occurs when drug and polymer concentrations are substantially increased. ${ }^{13-15}$ Furthermore, recent clinical data demonstrates ongoing reduction in luminal calibre beyond 6 to 8 months postintervention ${ }^{16}$ - the time point at which completion of vessel-wall healing was observed in the bare metal stent era. ${ }^{17}$ The common thread linking both late stent thrombosis and late erosion of luminal 
caliber ('late luminal creep') seems to be the existence of a persistent inflammatory response within the coronary vesselwall following polymer-coated DES implantation. ${ }^{16}$ A possible culprit appears to be the residual presence of permanent polymer following completion of its functional role..$^{2,5,18}$

\section{Bioabsorbable polymers}

Various strategies have been proposed to eliminate the potential harm associated with permanent polymer-based DES. One of these solutions is the use of bioabsorbable polymer. A recent study compared the healing and inflammatory responses of polymer-free bare-metal stents (BMS), polymer-free sirolimus-eluting stents (SES) and polymer-free sirolimus-eluting stents plus estradiol (SES + ED) to Cypher ${ }^{R}$ drug-eluting stents (CDES) in a rabbit model of overlapping stent placement. ${ }^{10}$ Twenty-eight rabbits received 2 overlapping stents in each iliac artery: SES, SES + ED, BMS, or CDES, and vessels were harvested at 28 days for histology and scanning electron microscopy. Although similar at nonoverlapping segments, neointimal thickness within the overlap site of CDES was significantly less than in SES, $\mathrm{SES}+\mathrm{ED}$, and BMS $(0.07 \pm 0.04 \mathrm{~mm}$ vs $0.16 \pm 0.03 \mathrm{~mm}$, $0.14 \pm 0.03 \mathrm{~mm}$, and $0.15 \pm 0.03 \mathrm{~mm}, P<0.0001)$. Endothelialization was greater in SES, SES + ED, and BMS compared with CDES in nonoverlapping sections $(80.0 \% \pm$ $5.0 \%$ vs $95.3 \% \pm 5.0 \%, 97.5 \% \pm 2.5 \%$, and $96.7 \% \pm 3.8 \%$; $P=0.0028)$ and overlapping sections $(85.8 \% \pm 2.9 \%$ vs $90.8 \%$ $\pm 6.3 \%, 89.2 \% \pm 6.3 \%$, and $48.3 \% \pm 2.9 \% ; P<0.0001)$. The number of luminal eosinophils was also less in overlapping sections of SES, SES + ED, and BMS versus CDES but was similar in nonoverlapping sections. The authors concluded that polymer-free stents coated with SES or SES + ED result in less robust neointimal suppression, but markedly improved arterial healing compared with CDES in the rabbit model. ${ }^{10}$

A number of biodegradable polymer-based DES have been tested in humans. One such device is the EXCEL sirolimus eluting stent (JW Medical Systems, China, Registration certification no. 2005DI3461514) which uses a novel polylactic acid (PLA) material. This material is gradually biodegraded within approximately 6 months, converting to water and carbon dioxide upon breakdown. The stent platform is a laser-cut, $316 \mathrm{~L}$ stainless steel, open cell design stent with strut thickness of 0.0047 inches. The PLA polymer is abluminally coated (only applied to the surface of the stent facing the arterial wall). Total sirolimus dosage varies from 195 to $376 \mu \mathrm{g}$ per stent according to the stent length and diameter, and is evenly mixed within the PLA coating.
In a study of 100 consecutive patients (153 lesions) receiving 6 months of dual-antiplatelet therapy treated at a single center, the EXCEL stent was associated with an encouragingly low $4.0 \%$ major adverse cardiovascular event rate at 12 months. ${ }^{19}$ During 24 months of follow up there were no cases of stent thrombosis, cardiac death or myocardial infarction. Quantitative coronary angiography at 12 months demonstrated 5.4\% in-segment restenosis (of which 3.6\% was in-stent). These preliminary data therefore provide encouraging initial safety and efficacy for this approach.

A much larger study with a biodegradable PLA polymer (BioMatrix Flex ${ }^{\circledR}$; Biosensors Inc, Newport Beach, CA, USA) used a biolimus-eluting stent versus a sirolimus-eluting stent with durable polymer (Cypher SELECT Cypher SELECT; Cordis, Miami Lakes, Fl, USA) has provided further encouraging data on the use of this novel polymer. ${ }^{20} 1707$ patients with chronic stable coronary disease or acute coronary syndromes were enrolled in a multicenter, assessor-blind, noninferiority study in 10 European centers. Biolimus-eluting stents were found to be noninferior for the primary endpoint (composite of cardiac death, MI or clinically driven target lesion revascularization) at 9 months (79 [9\%] patients vs 89 [11\%], rate ratio 0.88 [95\% CI 0.64-1.19], $P$ for non-inferiority $=0.003, P$ for superiority $=0.39)$. Frequency of cardiac death $(14[1.6 \%]$ vs 21 [2.5\%], p for superiority $=0.22)$, myocardial infarction (49 [5.7\%] vs 39 [4.6\%], $P=0.30$ ), and clinically-indicated target vessel revascularization (38 [4.4\%] vs 47 [5.5\%], $P=0.29$ ) were similar for both stent types. 168 (79\%) patients in the biolimuseluting group and 167 (78\%) in the sirolimus-eluting group had data for angiographic follow-up available. Biolimus-eluting stents were noninferior to sirolimus-eluting stents in in-stent percentage diameter stenosis $(20.9 \%$ vs $23.3 \%$, difference $-2.2 \%$ [95\% CI -6.0 to 1.6], $P$ for noninferiority $=0.001, P$ for superiority $=0.26$ ). The results indicate that a stent eluting biolimus from a biodegradable polymer represents a safe and effective alternative to a stent eluting sirolimus from a durable polymer in patients with chronic stable coronary artery disease or acute coronary syndromes. Nevertheless, the trial provides no insight as to whether the bioabsorbable polymer which characterizes this device will distinguish this biolimus-eluting stent from other stent platforms currently approved by the US Food and Drug Administration (FDA), in terms of increased safety (reduced rates of stent thrombosis) or shorter duration of dual antiplatelet therapy. Further studies will be required to address these issues with particular emphasis on longer follow up (significantly beyond the 9 months timeframe taken for the polymer to dissolve, during which time - in theory - greater benefit should accrue). 
In contrast to the encouraging data presented above, there have also been some notable failures in this arena. The MAHOROBA study examining a tacrolimus-eluting bioabsorbable polymer-coated cobalt chromium stent demonstrated failure of this device to prevent neointimal hyperplasia, with a composite MACE (major adverse cardiac event) rate of $23.4 \%$ among a pilot study of 47 patients. $^{21}$ Tests of a stent with a biodegradable polylactic-co-glycolic acid polymer releasing paclitaxel have shown this to be safe but less effective than an approved and widely used paclitaxel-eluting stent (TAXUS ${ }^{\circledR}$ ), presumably because of ineffective pharmacokinetics associated with the novel polymer. $^{22}$

Recent data examining the potential to reduce anti-proliferative drug dose in combination with a bioabsorbable polymer have indicated this may be another means of achieving greater safety without compromising efficacy. ${ }^{23}$ In a porcine study of low- and high-dose sirolimus release $(25,40$, or $100 \mu \mathrm{g})$ from hydroxyapatite (Hap) with Cypher (Cordis; Johnson \& Johnson, Warren, New Jersey, USA) (111 $\mu \mathrm{g}$ sirolimus), similar efficacy in prevention of neointimal hyperplasia was demonstrated, with increasing signs of delayed vascular healing in a dose-dependent manner. Cypher induced the highest amount of fibrinoid (considered to be a marker of delayed healing). ${ }^{23}$

\section{Polymer-free DES}

An alternative to use of a bioabsorbable polymer is complete avoidance of the use of polymer, applying the drug directly to the stent. Producing the same predictable and controlled release of drug over a sustained period is clearly more challenging without recourse to a polymer, but early human data of polymer-free DES have been encouraging.

One such study compared a novel polymer-free rapamycin- and probucol-eluting stent (Dual-DES) with a commercially available permanent polymer-based sirolimus-eluting stent (SES; Cypher) and with a zotarolimus-eluting (ZES; Endeavor) stent. ${ }^{16}$ This device utilizes a sand-blasting technique to generate micropores on the thin strut $(87 \mu \mathrm{m})$ stent surface which can then be directly coated using a mixture of the active compounds and a shellac resin $(0.07 \%)$ with no need for a polymer. A total of 1007 patients undergoing coronary stenting of de novo lesions, in native vessels, were randomized to treatment with SES ( $n=335)$, Dual-DES $(n=333)$, or ZES $(n=339)$. The primary endpoint was binary angiographic restenosis at 6- to 8-month follow-up angiography. Secondary endpoints were angiographic in-stent late loss; and target lesion revascularization (TLR), death/MI and stent thrombosis at 12 months. Follow-up angiographic data were available for $828(82.2 \%)$ patients.

There was a significant difference in both binary restenosis and TLR across treatment groups $(P=0.003$ and $P<0.001$, respectively). Binary restenosis in the Dual-DES group $(11.0 \%)$ was significantly lower than that in the ZES group $(19.3 \% ; P=0.002)$ but comparable with that in the SES group $(12.0 \% ; P=0.68)$. Similarly, TLR with Dual-DES (6.8\%) was significantly lower than ZES $(13.6 \% ; P=0.001)$ but not different to that of SES $(7.2 \% ; P=0.83)$. These differences were mirrored in the extent of late loss across the groups. No differences were observed between stent groups in terms of death/MI or stent thrombosis. These data support an approach to DES design that utilizes novel polymer-free drug dosing, while maintaining high antirestenotic efficacy. The potential of this approach to increase safety whilst reducing the need for prolonged dual antiplatelet therapy remains to be tested in larger cohorts however, with wider and more clinically relevant inclusion criteria. Such studies now being planned include the NEVO II trial. This randomized study will compare the $\mathrm{NEVO}^{\mathrm{TM}}$ Sirolimus-eluting Coronary Stent to the XIENCE V ${ }^{\mathrm{TM}}$ Everolimus-eluting Coronary Stent and aims to enroll 2000 patients. The NEVO stent utilizes a reservoir technology incorporating hundreds of small reservoirs, each acting as a depot into which drug(s) may be loaded.

\section{Bioabsorbable stent platforms}

An ambitious project to develop a fully biodegradable coronary stent is now underway. There are numerous theoretical advantages to such a device. The benefits of initial scaffolding to prevent acute recoil and treat dissection could still be maintained, but without the subsequent presence of a permanent prosthesis in the artery which may act as a substrate for late thrombotic events, or impede subsequent surgical revascularization. The potential to safely discontinue dual-antiplatelet therapy within a reasonable timeframe of several months postPCI is another enormous attraction. As noninvasive imaging techniques such as CT improve, the avoidance of a permanent metallic stent in the coronary artery (associated with blooming artifact) is also likely to be advantageous.

The technological challenge should not be underestimated. The required characteristics of sufficient mechanical strength to prevent negative vessel remodeling and avoid stent deformity/strut fractures, while still maintaining the capacity for full dissolution of the stent in a timely manner, will require tradeoffs between these competing aims. Whether such tradeoffs can actually be made in such as way as to improve 
overall safety and efficacy when compared to existing (or polymer-refined) DES is far from certain.

The first animal studies of biodegradable stents were performed in the late $1980 \mathrm{~s}$. Stack et $\mathrm{al}^{24}$ developed a prototype poly-L-lactide (PLA) stent that could withstand up to $1000 \mathrm{mmHg}$ of crush pressure. They designed the stent to maintain its radial strength for 1 month, with complete resorption of the device complete by 9 months post-implant. There were several reasons why this and similar biodegradable devices did not prosper and eventually succumbed to their bare-metal stent competitors. These included difficulties in manufacturing compatible biodegradable materials that had molecular weights capable of limiting inflammation and restenosis, ${ }^{25}$ concerns about loss of radial strength over time that increased the risk for stent fracture and migration of degraded products; ${ }^{26,27}$ and concerns about long-term biocompatibility of breakdown products. ${ }^{28}$ Finally, swelling of the biodegradable stent as it absorbs water to dissolve was also thought to exacerbate neointimal hyperplasia. ${ }^{29}$

Biodegradable stents that are currently in development fall broadly into one of two categories: those manufactured entirely from polymers versus devices that are made from bioabsorbable metals. ${ }^{30}$

\section{Polymer-based biodegradable stents}

Of the polymeric stents, greatest interest has focused on use of PLA as the polymer of choice. Polylactide polymers are already used in a variety of medical settings, including orthopedic prostheses and absorbable sutures. This polymer is metabolized through the Kreb's cycle (degraded to carbon dioxide and water over a period of 1-2 years) with small residual particles $<2 \mu \mathrm{m}$ in diameter capable of being phagocytosed by macrophages.

The Igaki-Tamai ${ }^{\circledR}$ stent (Igaki Medical Planning Co. Ltd., Kyoto, Japan) was the first PLA stent tested in humans. ${ }^{31}$ This stent was deployed using a thermal balloon expandable covered sheath system, which itself posed a number of potential problems. First, the heat required for rapid expansion $\left(65-75^{\circ} \mathrm{C}\right.$ for a few seconds) may produce arterial wall necrosis leading to proliferation of smooth muscle cells. ${ }^{32}$ Second, temperatures above $55^{\circ} \mathrm{C}$ increase platelet adhesion raising concerns regarding stent thrombosis. ${ }^{33}$ Initial experience however in a pilot study of 16 patients was encouraging, with late loss similar to that expected with a bare metal stent. ${ }^{31}$ In a larger study of 50 patients with 63 lesions (including lengths of $13.5 \mathrm{~mm}$ and a reference vessel diameter of $2.95 \mathrm{~mm}$ ) only one patient had a thrombotic event resulting in a Q-wave myocardial infarct at 1-year follow-up. ${ }^{30}$ In the same study, quantitative coronary angiography at 3, 6, and 12 months demonstrated percentage diameter stenoses of $12 \pm 8,38 \pm 23$, and $33 \pm 23$, respectively. Restenosis rates were $21 \%$ at 6 months and $19 \%$ at 12 months; the incidence of restenosis seemed to peak between 3 and 6 months, after which it decreased. The target lesion revascularization rate was $12 \%$ at 6 months and $17 \%$ at 12 months. There were no deaths or surgical revascularizations. At 4-year follow-up, $18 \%$ of the patients had repeat percutaneous coronary intervention (somewhat higher than seen with DES), there was one death, and the MACE-free event survival, as determined by a Kaplan Meier curve, was $82 \%$. Further animal studies have since been performed using drug-coated versions of this device which successfully limited restenosis, ${ }^{28,34}$ although human studies are still awaited.

Another device combining a PLA platform with the capability to elute everolimus has been developed by the Biosensors International company (Singapore). In this iteration, the PLA was cross-linked rather than linear (as in the Igaki-Tamai stent), a design feature thought to encourage preferential surface rather than bulk degredation. This should in theory ensure a more gradual change in the mechanical properties of the stent over time. Indeed, in animal models, there was no apparent loss in mechanical strength over the first 4 to 6 weeks following implantation. ${ }^{30}$

The only biodegradable drug-eluting PLA stent currently in clinical trials is the bioabsorbable everolimus-eluting coronary stent (BVS; Abbott Vascular, Santa Clara, CA, USA). This device has a backbone of circumferential hoops of highly crystalline PLA, with a strut thickness of $150 \mu \mathrm{m}$, linked directly together or held by straight bridges ${ }^{24}$ achieving a radial strength similar to the Multi-link Vision metal stent (Abbott Vascular). At both ends of the stent are two adjacent radio-opaque metal markers to facilitate correct fluoroscopic positioning. On the surface, there is a thin coating of a 1:1 mixture of everolimus $8.2 \mu \mathrm{g} / \mathrm{mm}$ and amorphous PLA matrix, allowing $80 \%$ of the drug to be released in 30 days. This is similar to the release patterns of the Xience $V^{\mathrm{TM}}$ (Abbott Vascular) and Cypher drug-eluting stents. Animal studies comparing the BVS PLA everolimus-eluting coronary stent with a control DES (Cypher) have shown that the inflammatory response is comparable at 6 and 9 months and significantly reduced at 12 months. ${ }^{35}$ There was complete luminal endothelialization by 28 days with a thin complete neointima composed of compact smooth muscle cells in a proteoglycan/collagen matrix overlying widely patent lumens.

Two year results from the ABSORB (a bioabsorbable everolimus-eluting coronary stent system) trial were 
recently published. ${ }^{36}$ In this study, 30 patients with a single de-novo coronary artery lesion were followed up for 2 years clinically and with multiple imaging methods: multislice CT, angiography, intravascular ultrasound, derived morphology parameters (virtual histology, palpography, and echogenicity), and optical coherence tomography (OCT). Clinical data were obtained from 29 of 30 patients. At 2 years, the device was safe with no cardiac deaths, ischaemia-driven target lesion revascularisations, or stent thromboses recorded, and only one MI (non-Q wave). 18-month multislice CT (assessed in 25 patients) showed a mean diameter stenosis of 19\% (SD 9). At 2-year angiography, the in-stent late loss of $0.48 \mathrm{~mm}$ (SD 0.28 ) and the diameter stenosis of $27 \%$ (11) did not differ from the findings at 6 months. The luminal area enlargement on OCT and intravascular ultrasound between 6 months and 2 years was due to a decrease in plaque size without change in vessel size. At 2 years, $34.5 \%$ of strut locations presented no discernible features by OCT, confirming decreases in echogenicity and in radiofrequency backscattering; the remaining apparent struts were fully apposed. Additionally, vasomotion occurred at the stented site and adjacent coronary artery in response to vasoactive agents indicating that more normal endothelial function was present. The authors concluded that at 2 years after implantation the stent was partially bioabsorbed, had vasomotion restored and restenosis prevented, and was clinically safe, suggesting freedom from late thrombosis. Although these data are encouraging, the small numbers of patients enrolled in the trial and the simplicity of the lesions treated means that a great deal of work remains to be done before these devices can be considered for use in routine clinical practice.

Another biodegradable PLA-based stent in development is an offspring of the Genous ${ }^{\mathrm{TM}}$ Bio-engineered cobalt-chromium R-stent ${ }^{\mathrm{TM}}$ (OrbusNeich, Fort Lauderdale, FL, USA). This device was designed to capture circulating endothelial progenitor cells (EPC) from the circulation by coating the surface of the stent with immobilized antibodies which bind to EPC-surface antigens. This approach should in theory lead to more rapid and complete endothelialization of the device. The bioabsorbable iteration replaces the metallic platform with a PLA-platform. Tantalum markers at both ends of the stent facilitate accurate placement under fluoroscopy. The abluminal surface is coated with a prohealing drug held in a bioabsorbable matrix, and the luminal side of the stent has the EPC-capturing antibodies. Incorporated within a hybrid-copolymer backbone is the antiproliferative drug with a programmable release profile. This stent is still in development and at this time no preclinical data have been published. ${ }^{30}$
A variation on the PLA-based design is the REVA stent (Boston Scientific Corporation, Natick, MA, USA). It is made from monomeric units of the common amino acid $L$-tyrosine, chemically modified to incorporate iodine molecules so as to make this stent radio-opaque. This polymer degrades to carbon dioxide and water in a similar fashion to PLA. It also forms ethanol. The stent struts are designed with a 'slide and lock' feature that enables strut thickness to be reduced without compromising radial integrity. Preclinical data have shown that at 30 days there is complete endothelialization of the bare REVA stent. ${ }^{30}$ Restenosis assessed by intravascular ultrasound was similar when compared to a bare metal stent at 12 months follow up. Interestingly, when compared to bare metal stents which displayed a relatively fixed luminal area over time, the REVA stent showed an increase in the luminal area from $3.65 \mathrm{~mm}^{2}$ at 1 month to $8.28 \mathrm{~mm}^{2}$ at 1 year. Boston Scientific is now developing a further evolution of this device which incorporates paclitaxel, whereby $50 \%$ of the antirestenotic drug will be eluted by day 10 , and $90 \%$ by 3 months. Enrollment in the RESORB trial (REVA Endovascular Study of a Bioabsorbable Coronary Stent) to test this device in 60 patients with short de novo lesions is expected to commence in late 2009.

Another device currently in development is the IDEAL ${ }^{\mathrm{TM}}$ stent (Bioabsorbable Therapeutics Inc., Menlo Park, CA, USA) which incorporates salicylic acid into the backbone of a polyanhydride ester (PAE) polymeric stent. The stent surface is coated with sirolimus, with compete elution of the drug by 30 days. The addition of salicylic acid in this device is designed to limit inflammation. When compared to bare metal stents, the PAE-salicylic acid polymer reduced angiographic restenosis at 30 day follow up ${ }^{30}$. Furthermore, the IDEAL stent reduced the inflammatory response and neointimal growth observed when compared to the Cypher stents. These preliminary findings from animal studies are encouraging and clinical data are eagerly awaited.

\section{Biodegradable metallic stents}

An absorbable metallic stent manufactured from magnesium has been developed by Biotronik (Berlin, Germany). This absorbable magnesium stent (AMS) is composed of 93\% magnesium and 7\% rare-earth metals laser cut from a single tube of alloy. It degrades within the body over a 2- to 3-month timeframe, forming inorganic salts containing calcium, chloride, oxide, sulfates and phosphates. The deposition of calcium within the vessel wall as a byproduct of magnesium degredation is clearly of some concern, and will be a particular focus of safety monitoring 
as this device is evaluated. The first generation stents had mechanical properties similar to current bare metal stents exhibiting high collapse pressure $(0.8$ bar $)$, low elastic recoil $(<8 \%)$ and minimum shortening after inflation $(<5 \%)$.

This device was first tested in the PROGRESS-AMS trial. ${ }^{37}$ A total of 63 patients (44 men; mean age 61.3) in 8 centers with single de novo lesions in a native coronary artery were enrolled in a multicenter, nonrandomized prospective study. Follow-up included coronary angiography and intravascular ultrasound at 4 months and clinical assessment at 6 months and 12 months. The primary endpoint was cardiac death, nonfatal MI, or clinically driven target lesion revascularization at 4 months. Among 63 patients a total of 71 stents were deployed, 10 to $15 \mathrm{~mm}$ in length and 3.0 to $3.5 \mathrm{~mm}$ in diameter. Diameter stenosis was reduced from 61.5 (SD 13.1\%) to $12.6(5.6 \%)$ with an acute gain of $1.41 \mathrm{~mm}(0.46 \mathrm{~mm})$ and in-stent late loss of $1.08 \mathrm{~mm}$ $(0.49 \mathrm{~mm})$. The ischemia-driven target lesion revascularization rate was $23.8 \%$ after 4 months, and the overall target lesion revascularization rate was $45 \%$ after 1 year. No MI, subacute or late thrombosis, or death occurred. Angiography at 4 months showed an increased diameter stenosis of 48.4 $(17.0 \%)$. After serial intravascular ultrasound examinations, only small remnants of the original struts were visible. This study demonstrated the feasibility of a magnesium-based bioabsorbable stent with acceptable safety and efficacy in this initial small and highly selected cohort.

Further evolution of the AMS device has targeted greater radial strength through modification of the magnesium-alloy composition. This AMS-2 stent should achieve a collapse pressure of 1.5 bar (compared to 0.8 with AMS-1), thereby limiting recoil to only $3 \%$. A drug-eluting version of this device is also now in development.

Iron has been proposed as an alternative metal to magnesium as a basis for bioabsorbable metallic stents. However, problems with the slow degredation time for iron-based devices (several years) and concerns regarding systemic iron-related toxicity have so far hampered progress with this approach.

\section{Conclusions and caveats}

There are a number of assumptions that underpin the hypothesis that bioabsorbable stents will lead to improved outcomes for patients when compared to a refined version of conventional DES. It is worth considering each of these in turn.

\section{Assumptions on dual antiplatelet therapy}

Currently, indefinite dual antiplatelet therapy is often routinely prescribed for all but the simplest DES procedures in the belief (or hope) that this approach reduces risk for late ischemic events, leading to better overall outcomes. It is assumed that by using a bioabsorbable stent the need for prolonged dual antiplatelet therapy will be eliminated with similar or better outcomes achieved.

It is important to emphasize that there are currently no data for or against the use of prolonged dual antiplatelet therapy (DAPT) in patients treated with DES. It is entirely possible that this approach produces the best outcomes for patients who require PCI, but not necessarily because of a reduction in stent thrombosis. It is possible that prolonged DAPT is an excellent way to treat high risk patients with coronary disease who have required intervention, regardless of stent-specific complications. Perhaps the benefits may extend to territories not treated with PCI or even to other vascular beds (such as in the prevention of stroke). If such data demonstrating a net benefit of prolonged DAPT on non-stent related outcomes were to emerge, then perhaps the biggest theoretical advantage of bioabsorbable DES would become irrelevant. Having the option of safely discontinuing DAPT in a patient who had received a bioabsorbable device would still remain as a potential advantage, but this too is an area where there remains tremendous uncertainty; an assumption that ischemic events following discontinuation of DAPT would be lower after use of a bioabsorbable stent should certainly not be taken for granted. Even among patients with acute coronary syndromes who are not treated with medical therapy alone (and no coronary stent) there is an excess of ischemic events after clopidogrel discontinuation. ${ }^{38}$

\section{Biodegradable $=$ biocompatible?}

It is worth emphasizing that a biodegradable polymer is not necessarily a biocompatible polymer. During degradation, polymers are broken down into molecules that are metabolized and removed from the body via normal metabolic pathways. In addition to these degradation products, many degradable materials release by-products that may reduce biocompatibility. PLA is an attractive polymer because its degradation involves hydrolysis of its ester bonds, which produces lactic acid, which is ultimately converted to water and lactic acid. However several studies have also suggested that PLA can produce an inflammatory response. ${ }^{25}$

Despite all the publicity, the fact remains that late stent thrombosis is a rare complication of conventional DES and by switching to bioabsorbable DES we may simply be replacing one mechanism for this rare complication with another. Calcification of the vessel wall during magnesium degradation remains an additional concern. 


\section{Incremental gains in the field of $\mathrm{PCl}$}

Advances in PCI over the past 2 decades have been remarkable. Although some obstacles and problems remain, overall the outcomes for an increasingly complex and aging population who are being treated with PCI are excellent. Even in these patients, the absolute specific frequency of following adverse events is low, requiring very large numbers of patients to document statistically significant and more important, statistically meaningful improvements in outcome with new device strategies. In the future, incremental advances will require increasingly greater effort for smaller and smaller rewards - this at a time when health budgets are coming under intense scrutiny and pressure.

Whereas previously there has been widespread application of very expensive new technologies across the spectrum of age and co-morbidities (often without proof of efficacy in groups excluded from randomized trials), it is not expected that in the future this approach will continue. Comparative efficacy studies of current (or polymer free) DES against bioabsorbable DES may demonstrate gains that are sufficiently small in absolute terms not to justify their widespread use in broad populations. It seems more likely that bioabsorbable DES may initially be used as a niche application, for example among patients who cannot take prolonged DAPT. As the elderly population of the western world grows however, and patients of increasing complexity are successfully treated with PCI, it is still reasonable to expect that niche markets of sufficient size will emerge to support the use and further development of exciting technologies such as this.

Perhaps the biggest question remaining is whether the presence of a permanent scaffold beyond a several-month period after PCI is indeed superfluous (as proponents of bioabsorbable stents contend). At the present time, this is something that cannot be answered by the available data. Once the initial technical challenges have been overcome (in particular balancing the need for radial strength against the ability of the device to fully dissolve) these questions can be addressed by large scale, randomized, controlled trials.

\section{Disclosures}

The authors declare no conflicts of interest.

\section{References}

1. Ako J, Morino Y, Honda Y, et al. Late incomplete stent apposition after sirolimus-eluting stent implantation: a serial intravascular ultrasound analysis. J Am Coll Cardiol. 2005;46(6):1002-1005.

2. Joner M, Finn AV, Farb A, et al. Pathology of drug-eluting stents in humans: delayed healing and late thrombotic risk. J Am Coll Cardiol. 2006;48(1):193-202.
3. Kotani J, Awata M, Nanto S, et al. Incomplete neointimal coverage of sirolimus-eluting stents: angioscopic findings. J Am Coll Cardiol. 2006;47(10):2108-2111.

4. Sousa JE, Serruys PW, Costa MA. New frontiers in cardiology: drugeluting stents: Part I. Circulation. 2003;107(17):2274-2279.

5. Virmani R, Guagliumi G, Farb A, et al. Localized hypersensitivity and late coronary thrombosis secondary to a sirolimus-eluting stent: should we be cautious? Circulation. 2004;109(6):701-705.

6. Camenzind E, Steg PG, Wijns W. Stent thrombosis late after implantation of first-generation drug-eluting stents: a cause for concern. Circulation. 2007;115(11):1440-1455; discussion 1455.

7. Pfisterer M, Brunner-La Rocca HP, Buser PT, et al. Late clinical events after clopidogrel discontinuation may limit the benefit of drug-eluting stents: an observational study of drug-eluting versus bare-metal stents. J Am Coll Cardiol. 2006;48(12):2584-2591.

8. Tsimikas S. Drug-eluting stents and late adverse clinical outcomes lessons learned, lessons awaited. J Am Coll Cardiol. 2006;47(10):2112-2115.

9. Doyle B, Rihal CS, O'Sullivan CJ, et al. Outcomes of stent thrombosis and restenosis during extended follow-up of patients treated with baremetal coronary stents. Circulation. 2007;116(21):2391-2398.

10. John MC, Wessely R, Kastrati A, et al. Differential healing responses in polymer- and nonpolymer-based sirolimus-eluting stents. JACC Cardiovasc Interv. 2008;1(5):535-544.

11. Carter AJ, Aggarwal M, Kopia GA, et al. Long-term effects of polymer-based, slow-release, sirolimus-eluting stents in a porcine coronary model. Cardiovasc Res. 2004;63(4):617-624.

12. Finn AV, Kolodgie FD, Harnek J, et al. Differential response of delayed healing and persistent inflammation at sites of overlapping sirolimus- or paclitaxel-eluting stents. Circulation. 2005;112(2):270-278.

13. Hoye A, Iakovou I, Ge L, et al. Long-term outcomes after stenting of bifurcation lesions with the "crush" technique: predictors of an adverse outcome. J Am Coll Cardiol. 2006;47(10):1949-1958.

14. Kuchulakanti PK, Chu WW, Torguson R, et al. Correlates and long-term outcomes of angiographically proven stent thrombosis with sirolimus- and paclitaxel-eluting stents. Circulation. 2006;113(8): 1108-1113.

15. Takano M, Ohba T, Inami S, Seimiya K, Sakai S, Mizuno K. Angioscopic differences in neointimal coverage and in persistence of thrombus between sirolimus-eluting stents and bare metal stents after a 6-month implantation. Eur Heart J. 2006;27(18):2189-2195.

16. Byrne RA, Mehilli J, Iijima R, et al. A polymer-free dual drug-eluting stent in patients with coronary artery disease: a randomized trial vs polymer-based drug-eluting stents. Eur Heart J. 2009;30(8):923-931.

17. Byrne RA, Iijima R, Mehilli J, et al. Durability of antirestenotic efficacy in drug-eluting stents with and without permanent polymer. JACC Cardiovasc Interv. 2009;2(4):291-299.

18. Finn AV, Nakazawa G, Joner M, et al. Vascular responses to drug eluting stents: importance of delayed healing. Arterioscler Thromb Vasc Biol. 2007;27(7):1500-1510.

19. Han Y, Jing Q, Chen X, et al. Long-term clinical, angiographic, and intravascular ultrasound outcomes of biodegradable polymercoated sirolimus-eluting stents. Catheter Cardiovasc Interv. 2008;72(2):177-183.

20. Windecker S, Serruys PW, Wandel S, et al. Biolimus-eluting stent with biodegradable polymer versus sirolimus-eluting stent with durable polymer for coronary revascularisation (LEADERS): a randomised non-inferiority trial. Lancet. 2008;372(9644):1163-1173.

21. Onuma Y, Serruys P, den Heijer P, et al. MAHOROBA, first-in-man study: 6-month results of a biodegradable polymer sustained release tacrolimus-eluting stent in de novo coronary stenoses. Eur Heart J. 2009 Jun;30(12):1477-1485.

22. Krucoff MW, Kereiakes DJ, Petersen JL, et al. A novel bioresorbable polymer paclitaxel-eluting stent for the treatment of single and multivessel coronary disease: primary results of the COSTAR (Cobalt Chromium Stent With Antiproliferative for Restenosis) II study. J Am Coll Cardiol. 2008;51(16):1543-1552. 
23. van der Giessen WJ, Sorop O, Serruys PW, Peters-Krabbendam I, van Beusekom HM. Lowering the dose of sirolimus, released from a nonpolymeric hydroxyapatite coated coronary stent, reduces signs of delayed healing. JACC Cardiovasc Interv. 2009;2(4): 284-290.

24. Stack RS, Califf RM, Phillips HR, et al. Interventional cardiac catheterization at Duke Medical Center. Am J Cardiol. 1988;62(10 Pt 2):3F-24F.

25. van der Giessen WJ, Lincoff AM, Schwartz RS, et al. Marked inflammatory sequelae to implantation of biodegradable and nonbiodegradable polymers in porcine coronary arteries. Circulation. 1996;94(7):1690-1697.

26. Venkatraman S, Poh TL, Vinalia T, Mak KH, Boey F. Collapse pressures of biodegradable stents. Biomaterials. 2003;24(12):2105-2111.

27. Uurto I, Juuti H, Parkkinen J, et al. Biodegradable self-expanding poly-L/D-lactic acid vascular stent: a pilot study in canine and porcine iliac arteries. J Endovasc Ther. 2004;11(6):712-718.

28. Vogt F, Stein A, Rettemeier G, et al. Long-term assessment of a novel biodegradable paclitaxel-eluting coronary polylactide stent. Eur Heart J. 2004;25(15):1330-1340.

29. Asplund B, Sperens J, Mathisen T, Hilborn J. Effects of hydrolysis on a new biodegradable co-polymer. J Biomater Sci Polym Ed. 2006;17(6):615-630.

30. Ramcharitar S, Serruys PW. Fully biodegradable coronary stents: progress to date. Am J Cardiovasc Drugs. 2008;8(5):305-314.
31. Tamai H, Igaki K, Kyo E, et al. Initial and 6-month results of biodegradable poly-1-lactic acid coronary stents in humans. Circulation. 2000;102(4):399-404.

32. Douek PC, Correa R, Neville R, et al. Dose-dependent smooth muscle cell proliferation induced by thermal injury with pulsed infrared lasers. Circulation. 1992;86(4):1249-1256.

33. Colombo A, Karvouni E. Biodegradable stents: "fulfilling the mission and stepping away". Circulation. 2000;102(4):371-373.

34. Yamawaki T, Shimokawa H, Kozai T, et al. Intramural delivery of a specific tyrosine kinase inhibitor with biodegradable stent suppresses the restenotic changes of the coronary artery in pigs in vivo. $\mathrm{J} \mathrm{Am} \mathrm{Coll}$ Cardiol. 1998;32(3):780-786.

35. Ormiston JA, Webster MW, Armstrong G. First-in-human implantation of a fully bioabsorbable drug-eluting stent: the BVS poly-L-lactic acid everolimus-eluting coronary stent. Catheter Cardiovasc Interv. 2007;69(1):128-131.

36. Serruys PW, Ormiston JA, Onuma Y, et al. A bioabsorbable everolimuseluting coronary stent system (ABSORB): 2-year outcomes and results from multiple imaging methods. Lancet. 2009;373(9667):897-910.

37. Erbel R, Di Mario C, Bartunek J, et al. Temporary scaffolding of coronary arteries with bioabsorbable magnesium stents: a prospective, nonrandomised multicentre trial. Lancet. 2007;369(9576):1869-1875.

38. Ho PM, Peterson ED, Wang L, et al. Incidence of death and acute myocardial infarction associated with stopping clopidogrel after acute coronary syndrome. Jama. 2008;299(5):532-539.
Medical Devices: Evidence and Research

\section{Publish your work in this journal}

Medical Devices: Evidence and Research is an international, peerreviewed, open access journal that focuses on the evidence, technology, research, and expert opinion supporting the use and application of medical devices in the diagnosis, treatment and management of clinical conditions and physiological processes. The identification of novel

\section{Dovepress}

devices and optimal use of existing devices which will lead to improved clinical outcomes and more effective patient management and safety is a key feature. The manuscript management system is completely online and includes a quick and fair peer-review system. Visit http://www. dovepress.com/testimonials.php to read real quotes from authors. 